\title{
Fortnightly Review
}

\section{Irritable bowel, irritable body, or irritable brain?}

\author{
Michael J G Farthing
}

This paper is based on the Lauran Harris lecture delivered by the author on 29 April 1993 at Boston University School of Medicine
The irritable bowel syndrome is probably the most common disorder encountered by gastroenterologists in the industrialised world and the most common functional bowel disorder seen by physicians in primary care. Most people with symptoms of the condition (60-75\%) do not consult a doctor. The cause of the irritable bowel syndrome is unknown, its development is poorly understood, and perhaps not surprisingly no universally agreed approach to treatment exists. Indeed it has been argued that controlled clinical trials have failed to show that any drug is effective in this condition. The irritable bowel syndrome is therefore a challenge for both doctors and patients, and important advances in treatment will probably not occur until the cause and development are better understood.

The irritable bowel syndrome, the most common of the functional bowel disorders (which form part of a wider range of functional gastrointestinal disorders), has recently been reclassified by Thompson et al. ${ }^{12}$ The condition is defined as a functional bowel disorder in which abdominal pain is associated with defaecation or a change in bowel habit, with the additional features of disordered defaecation and abdominal distension. The insistence that both abdominal pain and disordered bowel habit must be present may be too restrictive, and many gastroenterologists make a positive clinical diagnosis of the condition even if abdominal pain is only a minor, infrequent symptom or is absent, provided that the other characteristic features are present. Currently accepted definitions of functional gastrointestinal disorder, functional bowel disorder, and the irritable bowel syndrome are outlined in box 1 . Although different symptom complexes clearly exist that enable a symptom based classification to be made, no pathophysiological evidence exists that these are different conditions each with a fundamentally different cause and development; such a classification is vital, however, when patients are enrolled in research studies to maximise the possibilities for comparability between different centres.

\section{Diagnostic criteria}

In 1978 Manning et al described several abdominal symptoms that were more likely to be present in the irritable bowel syndrome than in organic abdominal disease (box 2). ${ }^{3}$ These symptoms were tested prospectively in subsequent studies and their validity confirmed. The more of these symptoms that are present, the more likely the patient is to have the irritable bowel syndrome. ${ }^{4}$ Kruis et al added other criteria, including a requirement for symptoms to have been present for more than two years and the use of symptom complexes that increase the chances of

Summary points
- The irritable bowel syndrome affects about
$20 \%$ of adults in the industrialised world
- The condition is a disturbance of
gastrointestinal function of unknown cause,
but abnormalities of intestinal motility and
visceral sensation are found in some patients
- Symptoms of the irritable bowel syndrome
are often associated with extraintestinal
manifestations, including urinary symptoms,
dyspareunia, and fatigue
- Overall, $40-60 \%$ of patients with the irritable
bowel syndrome who seek medical advice have
psychological symptoms of depression or
anxiety, or both
- Diagnosis of the irritable bowel syndrome
can usually be made on the basis of clinical
history and examination, but exclusion of
organic disease is advisable in patients aged
> 45 years who present with new bowel
symptoms
- No uniformly successful treatment exists for
the irritable bowel syndrome. End organ
treatment aimed at relieving abdominal pain
(antispasmodic drugs) or disturbed bowel
habit (antidiarrhoeal and bulking agents) can
produce symptomatic relief, and "central"
treatment (for example, anti-depressants,
hypnotherapy, psychotherapy) should be
considered in patients with associated affective
disorders

making a positive clinical diagnosis. ${ }^{5} \mathrm{~A}$ recent report produced clear cut diagnostic criteria for the irritable bowel syndrome, which are particularly valuable as entry criteria for research studies (box 3 )..$^{1}$

\section{Epidemiology}

Several surveys show that functional gastrointestinal symptoms occur in up to a fifth of adults in the industrialised world ${ }^{67} \mathrm{~A}$ recent community study found symptoms typical of irritable bowel syndrome in more than a fifth of women and a tenth of men and that prevalence was similar throughout life. ${ }^{7}$ Women seem to present more commonly than men to doctors with symptoms of the condition, although prevalence of symptoms in the community is similar in men and women. The irritable bowel syndrome is a transcultural disorder, with a similar prevalence reported in China, India, Japan, and South America, although prevalence 
Box 1-Functional disorders of the

gastrointestinal tract ${ }^{2}$

Functional gastrointestinal disorder

A variable combination of chronic or recurrent gastrointestinal symptoms not explained by structura or biochemical abnormalities. Some symptoms may be attributable to the oropharynx, oesophagus, stomach, biliary tree, small or large intestine, or anus

\section{Functional bowel disorder}

A functional gastrointestinal disorder with symptoms attributable to the middle or lower intestinal tract. The symptoms include abdominal pain, distension, and disordered defaecation

\section{Irritable bowel syndrome}

A functional bowel disorder in which abdominal pain is associated with defaecation or changes in bowel habit, and with features of disordered defaecation and with distension Africa. ${ }^{1}$ In the developing world symptoms of the irritable bowel syndrome might be more common in cities than rural areas. Only a minority of individuals with symptoms present to doctors. A recent survey of 854 employees of a single business community in the City of London showed that $16.6 \%$ had symptoms of the condition, but only a quarter of these people had consulted a doctor (J E Gomborone et al, unpublished observations).

The irritable bowel syndrome accounts for between a fifth and a half of referrals to gastroenterologists and thus represents a substantial workload for hospital specialists. In the United States the condition accounts for an estimated 2.4-3.5 million visits to physicians each year, and $2 \cdot 2$ million prescriptions are issued. Further financial implications result from days lost at work.

\section{Cause and development of the irritable bowel syndrome}

The failure to identify a specific cause of the irritable bowel syndrome or indeed to have a widely agreed understanding of its pathophysiological or psychophysiological processes makes it difficult to develop universally acceptable strategies for treatment. Several lines of investigation, however, have increased our understanding of the condition (box 4), although many would argue that the studies have raised as man questions as they have answered.
Box 2-
Symptoms more
likely to be found
in the irritable
bowel syndrome than organic
abdominal
disease $^{3}$
- Pain eased after bowel movement
- Looser stools at onset of pain
- More frequent bowel movements at onset of pain
- Abdominal distension
- Mucus in rectum
- Feeling of incomplete emptying

\section{A DISORDER OF MOTILITY OF THE GUT?}

The disturbance of bowel habit in the irritable bowel syndrome has led investigators for several decades to study several aspects of intestinal motility and transit. Initial studies focused on colonic motility using multiple, fine bore assemblies, initially perfused with water but more recently with solid state, pressure manometric catheters throughout the colon most studies have focused on the sigmoid colon and the rectum. Few clear cut differences have emerged between healthy control subjects and patients with the irritable bowel syndrome. ${ }^{8}$ Some studies suggest that basal colonic motility is normal whereas others show that it is increased, particularly in patients in whom constipation is the main symptom. Trotman and Misiewicz, however, showed that patients with the irritable bowel syndrome had lower colonic pressures may be lower in other parts of South East Asia and in

The reasons for the diversity in these findings probably relate to the selection of patients and methodological differences, including manometric techniques, bowel preparation, and sedation. When the colon is stimulated, however, by eating, balloon distension, or cholecystokinin, investigators agree that the colon of patients with the irritable bowel syndrome is hyperreactive. Some studies show that patients in whom constipation is the main symptom have greater responses than those in whom diarrhoea is the main symptom.

Thus although the findings of colonic motility in the irritable bowel syndrome differ widely, the colon of patients with the condition seems to be more sensitive to physiological and possibly pathophysiological stimuli. In all of these studies, however, a substantial overlap exists between the measurements in control subjects and those in the patients with the irritable bowel syndrome, indicating that no pathophysiological marker of the syndrome exists in the colon.

As studies of colonic motility failed to provide an immediate answer for the development of the irritable bowel syndrome investigators looked for motility disturbances in the small bowel. As with colonic motility, however, their findings differed. ${ }^{8}$ A poten-

\section{Box 3-Diagnostic criteria for the irritable bowel syndrome ${ }^{1}$}

At least three months of continuous or recurrent symptoms of:

- Abdominal pain or discomfort which is:

Relieved with defaecation and/or

Associated with change in frequency of stool and/or

Associated with a change in consistency of stool

- Two or more of the following, on at least a quarter of occasions or days:

Altered bowel frequency ( $>3$ bowel movements a day or $<3$ bowel movements a week)

Altered form of stool (lumpy/hard or loose/watery stool)

Altered passage of stool (straining, urgency, or feeling of incomplete evacuation)

Passage of mucus

Bloating or feeling of abdominal distension transducers. Because of the difficulties of placing than healthy control subjects and patients with diverticular disease. ${ }^{9}$ tially important new abnormality was detected during fasting motility-namely, "clustered contractions." These are short bursts of intense activity separated by long intervals of rest. Some studies incorporating clustered contractions occurred more often in patients with the irritable bowel syndrome than in healthy volunteers, although the fact that they occurred in individuals free of symptoms indicates that the contractions are not pathognomonic of the irritable bowel syndrome. Other studies, however, using similar methodologies, did not find any difference between patients with the irritable bowel syndrome and healthy volunteers. Some clustered contractions seem to coincide with episodes of abdominal pain. ${ }^{10}$ Manometry of the small intestine after meals has been studied, but again results differ in that some investigators believe that motility after meals is reduced in duration ${ }^{10}$ whereas others have found it to be normal. ${ }^{8}$ Acute stress has been shown to increase motility in the small bowel in some studies but inhibit it in others. ${ }^{8}$ Thus, although some evidence suggests that patients with the irritable bowel syndrome have abnormalities of motility in the small bowel, the heterogeneous findings make it difficult to put together a single, prolonged manometry of the small intestine found that 
Box 4-Possible factors in the development of the irritable bowel syndrome

- Disordered motility of the gut: Small intestine Colon

Oesophagus and stomach

- Altered gut sensation: Increased awareness of normal gastrointestinal events

Reduced sensation threshold for "pathophysiological" events

- Involvement of the central nervous system:

Altered central processing of end organ motor and sensory activity

Effect of mood on gastrointestinal function

unifying hypothesis for the cause and development of the irritable bowel syndrome.

The potentially important clinical correlate of intestinal motility-namely, intestinal transit-has also been studied. In cases of the irritable bowel syndrome in which diarrhoea is the main symptom both orocaecal transit time and whole gut transit time have been shown to be reduced, although again these findings are not universal. ${ }^{8}$ Diversity may well be accounted for by differences in the selection of patients and in measurement techniques. Although apparent abnormalities of motility in the small intestine and colon and transit time have been identified, many inconsistencies exist, and it is difficult to invoke a hypothesis that suggests that the irritable bowel syndrome is due to a primary motor disorder of the gut.

\section{A DISORDER OF SENSATION OF THE GUT?}

Debate continues on whether the irritable bowel syndrome is a condition in which abnormal motility is normally perceived or in which normal motility is abnormally perceived. Several studies have shown that increased sensitivity to balloon distension of the rectum exists in patients with the irritable bowe syndrome. ${ }^{112}$ This sensitivity is not part of a global, lower pain threshold as tolerance of cold and other painful stimuli produce either normal or reduced pain perception scores. ${ }^{13}$ Not all investigators, however, have been able to reproduce this increased rectal sensitivity. ${ }^{14}$ although others have been able to reproduce typical abdominal pain with balloon distension in the large or small intestine. ${ }^{15}$ Increased visceral sensation in patients with the irritable bowel syndrome may be a component of the disorder, ${ }^{16}$ although this is probably not the primary defect, partly because a substantial overlap exists between sensation thresholds of patients with the irritable bowel syndrome and those of healthy volunteers.

\section{PART OF AN IRRITABLE BODY?}

The evidence reviewed so far does not support the view that the irritable bowel syndrome involves a primary motility disorder or a primary disturbance of visceral sensation in the intestine. Additional information suggests that disruption of central control mechanisms that modulate the motility and sensation of the gut is more important than primary end organ dysfunction. Increasing evidence suggests that the symptoms of the irritable bowel syndrome are often accompanied by upper gastrointestinal symptoms such as nausea, vomiting, and early satiety; urinary symptoms such as nocturia, frequency, urgency, and the feeling of incomplete emptying of the bladder; and dyspareunia. ${ }^{1718}$ Evidence also suggests that extra- abdominal associations exist, including hyperreactivity of the bronchi. ${ }^{19}$ Smooth muscle is a common factor in all of these extraintestinal locations, and it seems logical, therefore, to invoke a hypothesis that involves disruption of central control rather than a primary, multiorgan hyperreactivity or hypersensitivity. Thus instead of merely an irritable bowel, there might also be an irritable oesophagus, an irritable stomach, an irritable bladder, and irritable vagina, and possibly irritable bronchi-or simply an irritable body.

\section{RESULT OF AN IRRITABLE BRAIN?}

Little doubt exists that emotional factors can alter the function of the gut. Any acute stress, such as an examination or an interview, can produce bowel frequency, nausea, vomiting, or early satiety. These symptoms suggest that acute, stressful events can affect the entire gastrointestinal tract, possibly with the patterns of symptoms being determined by individual susceptibility. It is also well recognised that urinary frequency commonly accompanies anxiety or acute, stressful events. Specifically, acute anger has been shown to increase the motility index in the colon, and acute stresses such as loud music and being woken from sleep can promote abnormalities of motility in the small intestine in patients with the irritable bowel syndrome that are different from responses in controls. ${ }^{8}$ Similarly the increase in the motility index in the colon associated with anger can be exaggerated in patients with the irritable bowel syndrome. Several studies have shown that immediately before presenting with the irritable bowel syndrome patients had a significantly increased stress score compared with healthy individuals. ${ }^{20}$

Hospital based studies have often shown an excess of psychological symptoms in patients with the irritable bowel syndrome compared with controls with organic gastrointestinal disease and healthy volunteers. Psychological symptoms include depression, anxiety, phobia, and somatisation. The patients seen in a hospital after referral either by a general practitioner or by another specialist may not be typical of those who remain in primary health care or do not consult a doctor at all. Whitehead et al studied subjects with the irritable bowel syndrome in the community and found that they had a similar psychological profile to healthy individuals without the condition..$^{21} \mathrm{~A}$ community study in which my colleagues and I compared the psychological profiles of consulters, non-consulters, and controls free of symptoms from the same population showed that the consulters with the irritable bowel syndrome had significantly higher anxiety and depression scores than either the non-consulters or the controls. ${ }^{22}$ Sixty per cent of the consulters fulfilled the diagnosis criteria of the Diagnostic and Statistical Manual of Mental Disorders, third edition, revised, including major depression (50\%), anxiety disorder $(43 \%)$, and dysthymia $(7 \%)$. However, the consulters judged their own pain to be more severe, frequent, prolonged, and disruptive to daily living than the nonconsulters did. But when the investigator blindly assessed the severity of symptoms in consulters and non-consulters he found no significant difference. These observations suggested that an individual's perception of his or her symptoms was important in determining whether he or she consulted a doctor. This finding was pursued further with questionnaires on attitude to illness, and consulters with the irritable bowel syndrome were clearly significantly more concerned about illness, particularly with respect to the subscales of disease phobia, hypochondriacal beliefs, and bodily preoccupation. ${ }^{23}$ Interestingly, the nonconsulters were also significantly different from the controls, with an intermediate position between these subjects and the consulters. 
A PRIMARY DISORDER OF THE PSYCHE?

The findings that a substantial proportion of consulters with the irritable bowel syndrome have a mood disorder and that non-consulters have different attitudes to illness from subjects who are symptom free indicate that central mechanisms may play a part in symptom production. Many patients who present with the irritable bowel syndrome do not have classic symptoms of an affective disorder, and mild depression can often be missed. In fact the alteration of affect may be different from standard psychiatric criteria, as my colleagues and I have shown in studies of recognition memory. ${ }^{24} \mathrm{~A}$ group of subjects with the irritable bowel syndrome, a group with depression, and a group of controls with organic disease were shown a series of positive, negative, and neutral words, and their memory was tested 30 minutes later to determine whether recognition memory was biased. As expected, the depressed patients recalled more negative words than the controls, but the patients with the irritable bowel syndrome were intermediate, which correlated well with their scores on the Beck depression inventory. However, the patients with the irritable bowel syndrome recalled substantially more negative words that were not included in the initial list than even the depressed patients. This finding seems to support the view that no clear parallel relation exists between depression scores and other measures of affect in patients with the irritable bowel syndrome.

\section{A psychosomatic model for the irritable bowel syndrome}

Evidence exists therefore of end organ dysfunction and possible abnormalities of central control of perception processing in consulters, and to a lesser extent in non-consulters, with the irritable bowel syndrome. No reproducible end organ pathophysiological or biochemical marker of the irritable bowel syndrome exists and the associated smooth muscle dysfunction or hypersensitivity in other parts of the body strongly suggests that central factors may have a primary role. Affective disorder may well determine whether a non-consulter becomes a consulter, but abnormal attitudes to illness may be present in nonconsulters with the irritable bowel syndrome which makes them more likely to seek medical advice even in the absence of affective disorder.

Not all patients with the irritable bowel syndrome have a clinical or subclinical affective disorder. Indeed many have normal bowel function until an episode of travellers' diarrhoea or food poisoning disrupts bowel function. Little if any controlled evidence exists that such events truly precipitate the symptoms of the irritable bowel syndrome, but anecdotal accounts and frequent reference to this link in standard textbooks suggests that it may be correct. The model therefore must include a somatic component that could be evoked by, for example, infection, dietary indiscretion, food intolerance but then for some reason continues as chronic dysfunction despite resolution of the primary result.

The question that remains unanswered is: What comes first, the chicken or the egg? Is it (a) the affective disorder or attitudes to illness that leads to the production of abdominal symptoms or to heightened awareness of mild and clinically unimportant abdominal symptoms that were previously disregarded or (b) the chronic abdominal symptoms that produce the affective disorder or disturbed perception, or both, by increasing concern about serious illness that propagates the syndrome?

Further investigation will no doubt continue to explore this conundrum. Treatment strategies cannot await the final characterisation of the process of the cause and development of the irritable bowel syndrome but must proceed on the information available, largely along symptomatic guidelines and the individual patient's requirements.

\section{An approach to management}

Gastroenterologists, general practitioners, and patients with the irritable bowel syndrome are under no illusions that no universally effective treatment exists for the condition. A review of controlled clinical trials of therapeutic agents in the irritable bowel syndrome reinforced this view and showed that most trials were flawed, and thus even those purporting to show therapeutic benefit should be questioned..$^{25}$ Clinicians who manage the condition tend to fall into two camps: (a) those who favour the end organ theory and $(b)$ those who favour the central approach, searching for and treating masked affective disorder and using psychological or behavioural approaches, or both, to stress management and symptom control (box 5). The two approaches are not mutually exclusive and can be used in sequence or combination, and indeed many clinicians and patients keep a foot in both camps.

\section{Box 5-Approaches to the management of the irritable bowel syndrome}

\section{- Consultation:}

Be positive in diagnosis of the irritable bowel syndrome

Avoid overinvestigation in young patients (under $40-45$ years)

- End organ treatment:

Explore dietary triggers

Give fibre for constipation

Give antidiarrhoeal drugs for bowel frequency

Give smooth muscle relaxants for pain

\section{- Central treatment:}

Physiological explanation of symptom production Counselling

Psychotherapy, hypnotherapy, cognitive therapy Antidepressants

\section{CONSULTATION}

The first consultation for a patient with the irritable bowel syndrome is critical. The time taken to explain possible mechanisms of symptom production, to reassure about fears of serious organic disease, and to explore potential social and psychological factors may have more long term benefit than any of the current drugs. The value of making a positive clinical diagnosis of the condition without resorting to exhaustive investigation to exclude an organic disorder has been stated many times.

\section{DIET}

Dietary modification is popular with patients, but other than the use of fibre in constipated patients, no firm recommendations can be given for specific dietary treatment. Some patients, however, are particularly sensitive to caffeine and foodstuffs that contain sorbitol, and withdrawal of these can often help. Similarly, other foods that seem to aggravate symptoms can be avoided, but highly complex exclusion diets have little to commend them in the treatment of the irritable bowel syndrome and may be counterproductive.

\section{END ORGAN TREATMENT}

Some patients with bowel frequency seem to benefit from an antidiarrhoeal preparation such as loperamide, 
and abdominal cramps in others are relieved by a smooth muscle relaxant. ${ }^{26}$ These agents are best used on a sporadic basis, as analgesics are used for a headache. The placebo response rate, however, is high in patients with the irritable bowel syndrome, ${ }^{25}$ and so several preparations may work in the short term.

\section{CENTRAL TREATMENT}

Real therapeutic triumphs in the irritable bowel syndrome can arise from treating mild or masked affective disorders. The clinician may be reluctant to enter this often difficult and time consuming arena and may feel poorly prepared to take an appropriate treatment to its conclusion, be it pharmacological, psychological, or a combination of the two. Clinical trials support the use of psychotherapy and hypnosis in the irritable bowel syndrome ${ }^{27-29}$ whereas trials of antidepressants are less impressive. ${ }^{25}$ However, antidepressants classically do not work in non-depressed patients, and thus correct selection of patients remains paramount. I and my colleagues have shown recently that antidepressants can alter intestinal motility and transit, and thus it may be helpful to take into account patterns of symptoms when choosing an antidepressant.

The tricyclic antidepressant imipramine slows intestinal transit and has inhibitory effects on manometry of the small bowel ${ }^{30}$ whereas the serotonin reuptake inhibitor paroxetine accelerates motility in the small intestine. ${ }^{31}$ Thus, although both drugs may have similar efficacy in treating depression, a patient in whom the main symptom is constipation may benefit most from a serotonin reuptake inhibitor, whereas a patient with bowel frequency may benefit from an antidepressant with anticholinergic properties. It should not be inferred from this central approach that affective disorder is the cause of the irritable bowel syndrome, but it is clearly an important reason why patients with symptoms of the irritable bowel syndrome seek medical advice and one that is often supremely amenable to treatment.

\section{Conclusion}

Our knowledge of the complex neural and neurohumoral communication systems between brain, mind, and gut is rudimentary and has proved difficult to study in humans. Nevertheless, it is within this network that the answers to the questions about the cause and development of the irritable bowel syndrome and related conditions lie. Methods for studying intestinal motor function, visceral sensation, and the central control of these in humans are relatively primitive, so we are unable to examine satisfactorily, for example, the direct relation between manometry, smooth muscle electrical activity, propulsive activity, and transit, all of which may be relevant in functional disorders of the gut. Even more challenging are the pathophysiological consequences in the gut of mood change and environmental stress.

Since the irritable bowel syndrome is so common it might be tempting to regard its consortium of symptoms as merely a variant of normality. For the individual with the condition this is unhelpful. We should use the limited knowledge that we have to provide realistic explanations for symptom production, accepting, for example, that we have no satisfactory explanation for the common occurrence of visible abdominal bloating.

Therapeutically, we need to consider: "Why is this person presenting with symptoms of the irritable bowel syndrome now? Is there evidence of an affective disorder?" This may be a more successful approach for relieving or reducing abdominal symptoms than end organ treatment. We must await new insights into the central and local control of intestinal motor and sensory function before the next important step forward is possible.

I am indebted to my colleagues Gerald Libby, Jennifer Gomborone, Paul Dewsnap, and David Gorard in the Psychosomatic Research Group at the Digestive Diseases Research Centre, Medical College of St Bartholomew's Hospital; the Priory Hospitals Group, which has supported much of the research of the group; and Mrs Shirley Harris for helpful discussion after the Lauran Harris lecture. Special thanks go to Nici Herrera for her help in preparing the manuscript.

1 Thompson WG, Creed F, Drossman DA, Heaton KW, Mazzacca G. Functional bowel disease and functional abdominal pain. Gastroenterology International 1992;5:75-91.

2 Thompson WG. Irritable bowel syndrome: pathogenesis and management. Lancet 1993;341:1569-72.

3 Manning AP, Thompson WG, Heaton KW, Morris AF. Towards positive diagnosis of the irritable bowel. $B M{ }^{\prime} 1978$;ii:653-4.

4 Thompson WG. Gastrointestinal symptoms in the irritable bowel compared with peptic ulcer and inflammatory bowel disease. Gut 1984;25:1089-92.

5 Kruis W, Thieme CH, Weinziel M, Schussler P, Hall J, Paulus WA, et al. A diagnostic score for the irritable bowel syndrome, its value in exclusion of organic disease. Gastroenterology 1984;87:1-7.

6 Jones R, Lydeard S. Irritable bowel syndrome in the general population. $B M$ 1992;304:87-90.

7 Heaton KW, O'Donnell LJD, Braddon FE, Hughes AO, Cripps PJ. Symptoms of irritable bowel syndrome in a British urban community: consulters and non-consulters. Gastroenterology 1992;102:1962-7.

8 Gorard DA, Farthing MJG. Intestinal motor function in irritable bowel syndrome. Dig Dis Sci 1994;12:72-84.

9 Trotman IF, Misiewicz JJ. Sigmoid motility in diverticular disease and the irritable bowel syndrome. Gut 1988;29:218-22.

10 Kellow JE, Gill RC, Wingate DL. Prolonged ambulant recordings of small bowel motility demonstrate abnormalities in the irritable bowel syndrome. Gastroenterology 1990;98:1208-18.

11 Prior A, Maxton DG, Whorwell PJ. Anorectal manometry in irritable bowel syndrome: differences between diarrhoea and constipation predominan patients. Gut 1990;31:458-62.

12 Whitehead WE, Holtkotter B, Enck P, Hoelzl R, Holmes KD, Anthony J, e al. Tolerance for rectosigmoid distension in irritable bowel syndrome. Gastroenterology 1990;98:1187-92.

13 Cook IJ, Van Eeden A, Collins SM. Patients with irritable bowel syndrome have greater pain tolerance than normal subjects. Gastroenterology 1987;93: 727-33.

14 Kendall GPN, Thompson DG, Day SJ, Lennard-Jones JE. Inter and intraindividual variations in pressure-volume relations of the rectum in normal subjects and patients with the irritable bowel syndrome. Gut 1990;31:1062-8.

15 Moriarty KJ, Dawson AM. Functional abdominal pain: further evidence that the whole gut is affected. $B M 71982 ; 284: 1670-2$

16 Mayer EA, Raybould HE. The role of visceral afferent mechanisms in functional bowel disorders. Gastroenterology 1990;99:1688-704.

17 Whorwell PJ, McCallum M, Creed FH, Roberts CT. Non-colonic features of irritable bowel syndrome. Gut 1986;27:37-40.

18 Talley NJ, Phillips SF, Bruce B, Zinsmeister AR, Wiltgen C, Melton LJ. Multisystem complaints in patients with the irritable bowel syndrome and functional dyspepsia. European fournal of Gastroenterology and Hepatology 1991;3:71-7.

19 White AM, Stevens WH, Upton AR, O'Byrne PM, Collins SM. Airway responsiveness to inhaled methacholine in patients with irritable bowel syndrome. Gastroenterology 1991;100:68-74.

20 Whitehead WE, Crowell MD, Robinson JC, Heller BR, Schuster MM. Effects of stressful life events on bowel symptoms: subjects with irritable bowel syndrome compared with subjects without bowel dysfunction. Gut 1992;33: 825-30.

21 Whitehead WE, Bosmajian L, Zonderman AB, Costa BJ, Schuster MM Symptoms of psychologic distress associated with irritable bowel syndrome: comparison of community and medical clinic samples. Gastroenterolog 1988;95:709-14.

22 Gomborone JE, Dewsnap PA, Libby GW, Farthing MJG. Community study reveals that dysfunctional illness attitudes in irritable bowel syndrome are not wholly a reflection of patient status. Gastroenterology 1993;104:A513.

23 Gomborone JE, Dewsnap PA, Libby GW, Farthing MJG. Abnormal illness attitudes in irritable bowel syndrome. $f$ Psychosom Res (in press).

24 Gomborone JE, Dewsnap PA, Libby GW, Farthing MJG. Selective affective biasing in recognition memory in the irritable bowel syndrome. Gu 1993;34:1230-3.

25 Klein KB. Controlled treatment trials in the irritable bowel syndrome: critique. Gastroenterology 1988;95:232-41.

26 Poynard T, Naveau S, Mory B, Chaput JC. Meta-analysis of smooth muscle relaxants in the treatment of irritable bowel syndrome. Aliment Pharmacol Ther 1994;8:499-510.

27 Whorwell PJ, Prior A, Colgan SM. Hypnotherapy in severe irritable bowel syndrome. Gut 1987;28:423-5.

28 Guthrie E, Creed F, Dawson D, Tomenson B. A controlled trial of psychological treatment for the irritable bowel syndrome. Gastroenterolog 1991;100:450-7.

29 Guthrie E, Creed F. The difficult patient: treating the mind and the gut European foumal of Gastroenterology and Hepatology 1994;6:489-94.

30 Gorard DA, Libby GW, Farthing MJG. Effect of a tricyclic antidepressant on small intestinal motility in health and in diarrhoea-predominant irritable bowel syndrome. Dig Dis Sci (in press).

31 Gorard DA, Libby GW, Farthing MJG. 5-Hydroxytryptamine and human small intestinal motility: effect of inhibiting 5-hydroxytryptamine uptake. Gut 1994;34:496-500.

(Accepted 8 Fuly 1994) 